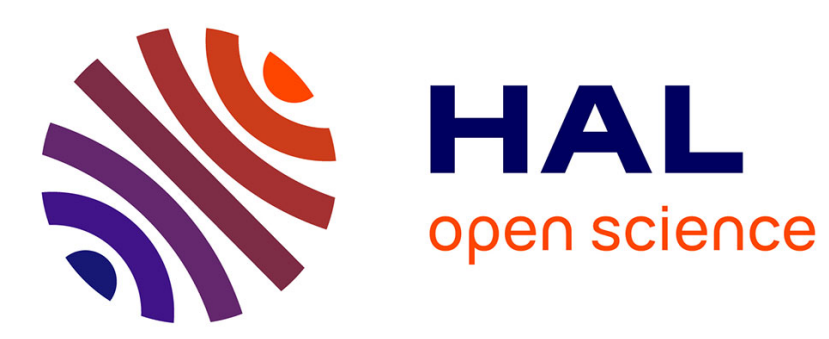

\title{
Exploration des terrae incognitae du savoir musical et construction identitaire en France (1842-1881)
}

\author{
Isabelle Mayaud
}

\section{To cite this version:}

Isabelle Mayaud. Exploration des terrae incognitae du savoir musical et construction identitaire en France (1842-1881). Cahiers Sens Public, 2016, 19-20, pp.194-210. 10.3917/csp.019.0194. hal01506838

\section{HAL Id: hal-01506838 \\ https://hal.parisnanterre.fr/hal-01506838}

Submitted on 20 Dec 2021

HAL is a multi-disciplinary open access archive for the deposit and dissemination of scientific research documents, whether they are published or not. The documents may come from teaching and research institutions in France or abroad, or from public or private research centers.
L'archive ouverte pluridisciplinaire HAL, est destinée au dépôt et à la diffusion de documents scientifiques de niveau recherche, publiés ou non, émanant des établissements d'enseignement et de recherche français ou étrangers, des laboratoires publics ou privés. 
Citation : Mayaud, Isabelle. «Exploration des terrae incognitae du savoir musical et construction identitaire en France (1842-1881), Circulation internationale des connaissances, Regards croisés sur la dynamique Nord-Sud, Cahiers Sens public, 2016, $\mathrm{n}^{\circ} 19-20$, p. $194-210$

DOI : $10.3917 /$ csp.019.0194

Me contacter : isabelmayaud@gmail.com 


\title{
Exploration des terrae incognitae du savoir musical et construction identitaire en France (1842-1881)
}

\author{
«Lointain se réfère dès l'ancien français à un plan spatial ou (1160) temporel. \\ [...] À la différence de l'adjectif, un, des lointains ont pris très tard leur sens \\ temporel (1863)» Rey, 2010,p. 1222)
}

Isabelle Mayaud $^{1}$

Motivée par la concurrence internationale, dans le domaine scientifique notamment, la France pose, dans la première moitié du xix ${ }^{\mathrm{e}}$ siècle, les jalons d'une activité culturelle ${ }^{2}$ à l'étranger. Cette politique publique passe par différents ministères et administrations d'État (Marine, Guerre, Affaires étrangères, Beaux-arts) ${ }^{3}$, et se déploie suivant des formes d'intervention diversifiées (missions individuelles ou collectives, temporaires ou permanentes, scientifiques et militaro-scientifiques) ${ }^{4}$. Fer de lance de cette action, le ministère de l'Instruction publique $^{5}$ compte à partir de 1842 , au sein de sa division des sciences et lettres, un « service des missions scientifiques et littéraires » en charge, en particulier, de l'évaluation et de la subvention de missions individuelles. Au fil des redécoupages administratifs, l'inscription institutionnelle de ce service évolue ${ }^{6}$, mais jusqu'en 1936 le ministère conserve un budget consacré aux missions (Bourquin, 1993, p. 17). "Système de voyages » (Ministère de l'Instruction publique, 1890, p. II), voué à la promotion raisonnée des mobilités savantes, son périmètre d'intervention s'étend aux confins du globe et ne souffre, en principe, aucune limite :

« La France, la première parmi les nations de l'Europe, avait commencé à envoyer, à travers le monde, des voyageurs intrépides et des savants distingués dont les découvertes enrichirent l'une après l'autre toutes les branches des connaissances humaines. [...] Cependant une direction générale manquait à ces efforts individuels » (Ministère de l'Instruction publique, 1890, p. I).

Dans le paysage de l'administration des circulations savantes en France au xix ${ }^{\mathrm{e}}$ siècle, les missions individuelles soutenues par le ministère de l'Instruction publique occupent une place singulière. Elles sont très nombreuses : Jean-Christophe Bourquin a recensé 2307 missions pour la période 1840-1914 (Bourquin, 1993, p. 122). Elles concernent divers champs de la connaissance : histoire, littérature, archéologie, géographie, philosophie, histoire naturelle, histoire de l'art, astronomie, géologie, etc. Elles mobilisent une pluralité d'outils et de méthodes

\footnotetext{
${ }^{1}$ Étudiante de doctorat à l'École des hautes études en sciences sociales (Centre Georg Simmel), Université Paris 8 (LabTop), sous la direction de Michael Werner et Laurent Jeanpierre. Sa thèse porte sur la production de savoirs sur les lointains musicaux (France, 1850-1920).

2 À mi-chemin entre la « diplomatie culturelle » et « l'action culturelle à l'étranger », telles que définies par Dominique Trimbur (Dubosclard, Grison, Jeanpierre, 2002, p. 18) - le distinguo résidant dans l'impulsion de départ : officielle pour la première, privée pour la seconde - cette politique se fonde sur une mise en correspondance de l'initiative publique avec les initiatives privées.

${ }^{3}$ Sur les multiples agents intervenant dans la régulation du voyage savant au xix ${ }^{\mathrm{e}}$ siècle, voir : Venayre, 2012 , pp. 215-250, chap. VII. Le prestige de la science.

4 À titre exemplaire, on renvoie à l'expédition d'Égypte (1798-1801), première grande initiative militaroscientifique et à l'ouverture du premier établissement à caractère scientifique et politique à l'étranger : l'École française d'Athènes en 1846.

${ }^{5}$ Le ministère de l'Instruction publique est rebaptisé ministère de l'Éducation nationale en 1932.

${ }^{6}$ Sur la généalogie de ce service et pour un inventaire succinct de ses archives, voir la notice de Marie-Élisabeth Antoine (Antoine, 1977).
} 
de recherche (archive, bibliothèque, terrain, etc.). Enfin, contrairement aux missions pérennes ou aux expéditions collectives, les missions individuelles ne se heurtent, a priori, à aucune limite géographique.

Le service des missions a fait l'objet d'études ${ }^{7}$ qui se fondent principalement sur les archives institutionnelles ${ }^{8}$ aujourd'hui conservées aux Archives nationales (France, site de Pierrefitte-sur-Seine), dans la sous- série $\mathrm{F}^{17}$, fonds du ministère de l'Instruction publique (fin $\mathrm{xvii}^{\mathrm{e}}-\mathrm{xx} \mathrm{e}^{\mathrm{e}}$ siècles). Ces archives sont lacunaires, mais pour le $\mathrm{xix} \mathrm{e}^{\mathrm{e}}$ siècle, les dossiers individuels sont bien conservés (Bourquin, 1993, p. 18). À partir de 1850, paraissent en outre, de manière épisodique, les Archives des missions scientifiques et littéraires qui reproduisent certains des rapports de mission adressés au ministre ${ }^{9}$. Les études d'histoire de la musique au xix ${ }^{\mathrm{e}}$ siècle n'ignorent pas l'existence de ces missions, souvent mentionnées à titre indicatif dans les biographies de savants. Les missions « musique »n'ont toutefois jamais fait l'objet d'une étude systématique. À partir des instruments de recherche ${ }^{10}$, de la consultation des dossiers individuels ${ }^{11}$ et du dépouillement des revues imprimées (voir note 9), on peut identifier 21 demandes (19 acceptées $^{12}$ ) de missions « musique » pour la période 1842-1881 (voir tabl. 1). Cet échantillon comprend exclusivement les missions expressément motivées par des recherches portant sur l'objet «musique ». Le cadre temporel reprend, quant à lui, le découpage chronologique opéré par Jean-Christophe Bourquin : « les missions se dirigent surtout vers la France et l'Europe » durant cette période (Bourquin, 1993, p. 161).

Des obstacles économiques ou techniques ont pu être invoqués pour expliquer l'orientation géographique, relativement limitée, des missions entre 1842 et 1881 . L'étude micro menée à partir de la vingtaine de missions « musique » permet, sinon de remettre en cause ces analyses, du moins de les enrichir à travers d'autres pistes, spécifiques aux conditions de la production de savoirs en France au xix ${ }^{\mathrm{e}}$ siècle. Un premier temps du développement permettra ainsi de prendre la mesure de l'emprise inédite de l'État-nation français, via son administration, sur les mobilités savantes. Un second temps déterminera les contours du savoir produit, à l'aune de contraintes traditionnellement identifiées et d'une autre variable peu explorée par les études des sciences, ressortant d'un « inconscient culturel national » (Bourdieu, 2002, p. 7) qui entraverait, c'est l'hypothèse qui sera développée, l'accès à la connaissance de l'altérité musicale.

\footnotetext{
${ }^{7}$ Les historiens Pascal Riviale (1991) et Jean-Christophe Bourquin (1993) ont consacré au service des missions une part significative de leurs travaux. À la faveur d'un investissement des archivistes (Le Goff, 2010, 2011) et du développement des études d'histoire culturelle, ce service bénéficie depuis peu d'un regain d'intérêt (voir notamment : Wiener, 2010 et la thèse en cours de Pierrette Chapelle). La présente étude s'inscrit dans le sillage de ces recherches.

${ }^{8}$ Aux ressources directement fournies par le fonctionnement du service s'ajoutent des archives éparses, disséminées entre les fonds publics et privés, autant de traces des trajectoires originales des différents chargés de mission.

${ }^{9}$ Les rapports des chargés de mission publiés par l'administration avant 1849 figurent dans le Journal général de l'instruction publique. Les Archives des missions scientifiques et littéraires paraissent ensuite de manière irrégulière entre 1850 et 1912 (pour le détail des livraisons, voir : Bourquin, 1993, p. 73). Quelques rapports de mission sont imprimés dans la Revue des Sociétés savantes entre 1860 et 1864.

${ }^{10}$ Les missions ont fait l'objet d'un répertoire numérique (Antoine, Olivier, 1975-1981), récemment complété par un index nominatif des voyageurs et un index géographique des missions (Le Goff, 2005).

${ }^{11}$ Les dossiers individuels des missions scientifiques et littéraires consultés pour cette étude sont compris entre les cotes $\mathrm{F}^{17} 2933-\mathrm{F}^{17} 3014^{2}$ qui couvrent principalement des voyages effectués au xix $\mathrm{e}^{\mathrm{s}}$ siècle.

${ }^{12}$ Les refus étaient généralement rares (Bourquin, 1993, p. 130).
} 


\section{Le service des missions : un avant-poste frontière}

\section{Administrer des instructions}

De la «peregrinatio academica » au « grand tour », une longue tradition confond, en Europe, voyage et savoir ${ }^{13}$. La volonté de contrôle de l'un par l'autre se traduit en France, à la fin du xviie siècle, par l'apparition des instructions aux voyageurs spécifiquement destinées à encadrer leurs observations (Bourguet, 1997, p. 1093). Depuis l'époque moderne, divers organes, publics et privés, prennent ainsi en charge la régulation des déplacements savants (Bourguet, 1996 ; Blanckaert, 1996) ; en ce sens, l'action de l'administration publique en France au xix ${ }^{\mathrm{e}}$ siècle n'est pas singulière. Elle se distingue toutefois de par son ampleur et son caractère systématique. Le ministère de l'Instruction publique, en concurrence avec d'autres administrations, des institutions d'État et des sociétés savantes, multiplie les initiatives. Pour le

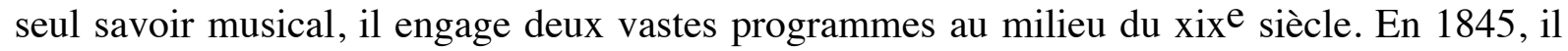
institue une «Commission des chants religieux et historiques de la France » et en 1852 il lance l'enquête dite Fortoul ${ }^{14}$. Ces projets, qui ne concernent que l'espace national, interviennent concurremment à la démarche d'Arcisse de Caumont (1801-1873), notable normand qui fonde en 1839 l'Institut des provinces afin d'encadrer les études entreprises par les érudits locaux (Guillet, 2003, p. 100). Ils attestent d'une volonté politique de contrôle des connaissances produites sur le territoire national, qui passe par la coordination des initiatives individuelles localisées, à la fois en amont (délivrance d'instructions ${ }^{15}$ ) et en aval (centralisation des études à des fins éditoriales $\left.{ }^{16}\right)$.

\section{Le cycle de vie des missions}

Ces deux enquêtes sont contemporaines du développement du service des missions qui participe d'une même politique publique de promotion des études savantes. Le « cycle de vie des missions »(Le Goff, 2011, p. 168) est connu : les dossiers individuels conservés aux Archives nationales portent en effet l'empreinte d'une procédure normalisée. Quel que soit le domaine de savoir visé, être " chargé de mission » induit l'acceptation de se soumettre à un parcours institutionnel. Les pièces délivrées par les demandeurs sont invariablement les mêmes (mais pas toujours toutes présentes) : une lettre de motivation, une liste de publication, une biographie succincte et une ou plusieurs lettres de recommandation. Suivent ensuite diverses pièces relatives à l'évaluation de la demande : elles émanent principalement de l'Institut et, à partir de 1874, de la Commission des missions. Lorsque la mission est acceptée, un « arrêté de mission » désigne officiellement un chargé de mission, précise la tâche à accomplir, éventuellement la durée de la mission et indique, le cas échéant, l'indemnité allouée. Un certain nombre de résultats étaient explicitement attendus. Au retour, les missionnaires, dans l'idéal,

\footnotetext{
${ }^{13}$ Sur l'articulation entre voyage et formation, voir : Roche, 2003 et Wagner, 2007.

${ }^{14} \mathrm{Du}$ nom du ministre de l'Instruction publique de l'époque, Hippolyte Fortoul (1811-1856); il s'agit d'un vaste recueil de chants et poésies populaires engagé dans les campagnes françaises entre 1852 et 1857.

15 Jean-Jacques Ampère (1800-1864) rédigea les instructions de l'enquête Fortoul, en limitant la collecte aux frontières politiques (et non linguistiques) de la nation (Thiesse, 2001, p. 171).

${ }^{16}$ Le projet d'édition, dans le cadre de l'enquête Fortoul, ne vit jamais le jour, mais plusieurs études parurent isolément.
} 
publiaient leurs rapports, présentaient les objets qu'ils rapportaient lors d'expositions, pour les céder ensuite aux musées ${ }^{17}$, et déposaient les livres collectés à la bibliothèque nationale.

\section{Les conditions du soutien public}

Expressément recherché par les demandeurs, l'appui du ministère offrait de multiples avantages. Financiers : les subsides consentis pour notre échantillon représentaient un complément de salaire apprécié et se cumulaient avec des facilités de transport (octroi d'une remise de $30 \%$ par les compagnies maritimes et gratuité des voyages à bord des navires de guerres ; Riviale, 1996, p. 70-71). Être «chargé de mission » donnait accès au circuit et aux ressources diplomatiques : Louis-Albert Bourgault-Ducoudray est introduit en Grèce par des lettres de recommandation adressées au ministre de France à Athènes et aux consuls en poste à Corfou et à $\mathrm{Syra}^{18}$. Une mission officielle permettait à un fonctionnaire de se libérer de ses obligations : Auguste Dozon est temporairement déchargé de son activité de vice-consul de France $^{19}$. Le soutien du ministère était enfin convoité pour le prestige qu'il conférait : le père de François-Anatole Laurent-de- Rillié intercède ainsi en faveur de son fils alors que celui-ci effectue déjà des recherches à Madrid ${ }^{20}$, afin d'obtenir une valorisation symbolique de ses recherches via l'estampillage «mission du ministère ». Indéniables, tous ces bénéfices n'en demeuraient pas moins conditionnés. Conçu à des fins de régulation des circulations savantes dans un souci d' « avantage public et d'utilité nationale $»^{21}$, le service des missions est structurellement doté d'un pouvoir d'autorité sur les scientifiques et leurs productions. Il détermine ce qui mérite étude et ce qui peut être édité : le fonds recèle ainsi des lettres de soutien destinées à valider les résultats et encourager les publications ${ }^{22}$ ou a contrario les proscrire ${ }^{23}$. Les objets collectés le temps de la mission lui appartiennent, du moins en principe. Ce transfert de biens, du chargé de mission au domaine public, n'allait en réalité pas de soi au milieu du $\mathrm{xix}^{\mathrm{e}}$ siècle : Théodore Nisard dénonce ainsi Félix Danjou pour avoir fait sien « des trésors qui reviennent de droit à l'étude publique $»^{24}$, en vendant à l'éditeur Blanchet des livres glanés au cours de sa mission italienne.

Organe d'accréditation et de financement, le service des missions participe donc d'une politique publique d'encadrement des déplacements. Point de passage obligé, ce service constitue un poste d'immobilité - corollaire des mobilités - (Latour, 2009) qui contraint les

\footnotetext{
${ }^{17}$ L'ouverture au public du Musée d'ethnographie du Trocadéro en 1879 correspond à une volonté politique de rassembler et exposer les objets collectés lors des missions en un lieu permanent. Sur l'histoire de ce musée, voir : Dias, 1991.

${ }^{18}$ Arch. nat., $\mathrm{F}^{17}$ 2971. Lettre du directeur des consulats et affaires commerciales du ministère des Affaires étrangères, Jean-Louis Meurand, au ministre de l'Instruction publique, Arthur de Cumont, Paris, 26 septembre 1874.

${ }^{19}$ Arch. nat., $\mathrm{F}^{17} 2956^{2}$. Dossier Dozon.

${ }^{20}$ Arch. nat., $\mathrm{F}^{17} 2981^{2}$. Dossier Laurent-de-Rillié.

${ }^{21}$ Arrêté du 30 janvier 1850. Voir aux Archives nationales le Bulletin universitaire contenant les ordonnances, règlements et arrêtés concernant l'Instruction publique (puis Bulletin administratif), conservé dans la sous-série AD/XIXh (1789-1943).

${ }^{22}$ Arch. nat., $\mathrm{F}^{17} 2956^{2}$. Dossier Dozon. Lettre du directeur de l'école française d'Athènes, Émile Burnouf, au ministre de l'Instruction publique, Saint-René Taillandier, Athènes, 18 juillet 1872.

${ }^{23}$ Arch. nat., $\mathrm{F}^{17} 2995^{1}$. Lettre de Théodore Nisard à François Génin, chef de division au ministère de l'Instruction publique, Grenelle, 2 décembre 1850.

${ }^{24}$ Ibid.
} 
circulations individuelles et détermine les contours des connaissances produites ; la normalisation des processus de validation induisant de facto une homogénéisation des savoirs.

\section{À la recherche des lointains perdus}

\section{L'orientation géographique des missions « musique »}

Observée pour l'ensemble des missions, l'orientation européenne des voyages se confirme pour les études musicales ${ }^{25}$. Durant la période 1842-1881, 7 chargés de mission se déplacent en France, 9 en Europe dans des pays limitrophes de l'hexagone, et 2, à la fin de la période, dans des pays européens non limitrophes. Cet éloignement relatif est imputable aux financements. Le budget du service des missions passe en 1843 de 12000 à $112000 \mathrm{fr}$, il chute à $66000 \mathrm{fr}$ en 1845, $25000 \mathrm{fr}$ en 1857, puis il remonte : $75000 \mathrm{fr}$ en 1863 et $150500 \mathrm{fr}$ en 1877 (Riviale, 1996, pp. 82-83). Les mutations en matière de transports et de communications sont également déterminantes. Différentes thèses s'opposent quant à la qualification (" révolution » ou « évolution »?) et la datation de ces transformations (Caron, 1997; Studeny, 1995, 200926); mais l'on convient que le second Empire marque une étape majeure dans le développement des chemins de fer et l'avènement du « règne de la vitesse » (Studeny, 1995). Ces différents facteurs, économiques, techniques et logistiques, constituent ainsi des éléments d'explication incontournables. Ils demeurent toutefois insuffisants pour saisir les ressorts des mobilités savantes. Suivant Jean-Christophe Bourquin, les missions soutenues durant la période 1882-1914 sont plus souvent exotiques, la nouvelle orientation participant d'une politique impérialiste alors menée par la France (Bourquin, 1993, p. 131). Ces desseins impériaux sont relativement étrangers aux agents de la période antérieure, pour autant, des logiques politiques président aussi à la validation des déplacements physiques et à la promotion de certains champs de recherche.

\section{L'horizon français des études musicales}

$\mathrm{La}$ « nationalisation de la vie intellectuelle au xix $\mathrm{e}$ siècle est un fait incontestable » (Sapiro, 2009, p. 11) : elle se traduit par le développement d'institutions localisées, mais aussi, ce qui est moins connu, par la formation de traditions nationales savantes (Heilbron, 2008, p. 8). L'étude micro- historique des missions «musique » pose la question du contenu de la production savante en ces termes et permet d'envisager les frontières bornant les savoirs non pas à l'aune de la seule variable « destination géographique », mais dans un cadre analytique élargi, intégrant le processus de construction culturelle nationale à la réflexion. En termes de champ d'étude spatial, les missions se répartissent comme suit : 12 chargés de mission travaillent sur la France, 2 sur l'Europe limitrophe (Italie et Espagne en tout début de période), 3 sur l'Europe non limitrophe (Macédoine et Grèce en fin de période), enfin 2 sur l'Europe en général. La France constitue donc le principal objet d'étude de ces missions, ceci quelle que soit leur destination. Cette orientation induit un certain type de recherche : les investigations

\footnotetext{
${ }^{25}$ L'analyse qui suit considère les seules missions acceptées, à l'exception de celle accordée à Paul Scudo, les informations la concernant étant incomplètes, ce qui porte l'échantillon à 18 missions (voir Tabl. 1).

${ }^{26}$ La bibliographie sur l'histoire des moyens de transport au xix ${ }^{\mathrm{e}}$ siècle est immense, pour un état récent de la question, voir Venayre, 2012, pp. 31-72, chap. I. Le triomphe de la communication.
} 
sur la France, menées dans les autres pays européens, se font principalement en bibliothèque. Le profil des savants est quant à lui socialement non homogène, mais les 16 chargés de mission identifiés partagent au moins trois caractères. Certains œuvrent dans des domaines en lien avec la musique : la critique (Scudo), la direction de presse (Danjou), la théorie (Laurent-de-Rillié), l'écriture de chants (Luzel, Quellien). D'autres exercent des fonctions de professeur (Clément, Bourgault-Ducoudray), de bibliothécaire (Meyer, Ruelle) ou d'archiviste (Bonnardot). On compte également deux religieux (Nisard, Touroude), un avocat (Baecker), un médecin (Arbaud) un diplomate (Dozon) et un aristocrate (Hersart de la Villemarqué). Tous ces individus sont donc des hommes, lettrés, dont la recherche sur la musique ne constitue pas l'activité principale.

\section{Les lointains de l'intérieur}

Si du double point de vue de l'orientation géographique et de l'objet d'étude, les missions «musique » sont relativement proches, elles gagnent en distance en termes de champ d'étude temporel. Cette dernière variable apparaît centrale pour comprendre les études musicales au xix ${ }^{\mathrm{e}}$ siècle. Le Haut Moyen-Âge et le Moyen-Âge représentent les périodes historiques de prédilection des chargés de mission : la musique en France s'inscrit dans une longue tradition que l'on fait remonter jusque Charlemagne. Au fil des recherches s'élabore ainsi une histoire de la musique européenne. Celle-ci repose sur deux socles inébranlables : la chrétienté (de nombreuses études portent sur les origines du chant grégorien) et, secondairement, l'Antiquité (la focale temporelle s'élargit vers la Grèce à partir des années 1870). La période contemporaine, quant à elle, n'est pas absente du corpus, mais elle concerne exclusivement les missions de collecte de musique populaire. Le lointain est donc ici social, mais également temporel. De fait, le concept de « primitif de l'intérieur » a été empiriquement bien développé, dans le cadre, notamment, des travaux conduits sur l'enquête Fortoul (voir note 13, supra) et récemment théorisé (Laborde, 2009 et Fabre, Privat, 2010). Dans son rapport sur la musique espagnole, Laurent de Rillé explique ainsi pourquoi les musiques populaires n'ont pas évolué :

« Dans chaque pays ce qu'on appelle spécialement le peuple, c'est-à-dire les classes ouvrières et agricoles, plus, en Espagne, la classe des pasteurs, n'ayant pas, comme les classes élevées et riches, la facilité de communiquer avec les nations voisines, au moyen des voyages, des relations de commerce, ou des alliances de famille, garde les usages, les costumes et toutes les traditions nationales, longtemps après que la noblesse ou la bourgeoisie ont usé leurs angles originaux par le frottement avec les étrangers. »

Les paysans bretons à la rencontre desquels se rendent Luzel ou Quellien sont donc les dépositaires des précieux vestiges encore vivants, comme figés hors du temps, d'un lointain passé musical de la nation.

En même temps que se construit une histoire diachronique de la musique européenne, se dessine donc une histoire synchronique de la musique des petites patries françaises, qui participe de la fabrique de stéréotypes culturels régionaux constitutifs de la nation ${ }^{27}$. Les nouvelles nations s'imaginent d'emblée comme anciennes (Anderson, 1983, p. 14) ; la distance temporelle intervenant ici comme un point de rencontre des différentes études musicales.

\footnotetext{
${ }^{27}$ Sur la fabrique culturelle de la Bretagne, voir Bertho, 1980. Pour des analyses similaires sur d'autres régions voir la note 3, p. 89 dans Guillet, 2003.
} 
Ainsi s'intitule la nouvelle de Dino Buzzatti qui narre le parcours hasardeux d'une femme à travers le monde. Dans cette fiction, l'héroïne quitte son village natal pour aller de ville en ville ; et, au terme d'un très long périple, se retrouver finalement dans ce même petit village... Proche et lointain se déclinent ici dans l'espace et le temps et s'articulent à la petite patrie originelle.

Les structures de la mobilité savante évoluent en France au milieu du xix ${ }^{\mathrm{e}}$ siècle vers une centralisation administrative renforcée et un déploiement international, qui passe, notamment, par le service des missions du ministère de l'instruction publique. Organe institutionnel positionné à la croisée de l'action et de la diplomatie culturelle, son étude permet d'enrichir l'histoire de la genèse des relations culturelles de la France à l'étranger.

Le suivi du parcours administratif conduit à étoffer la thèse du centralisme « à la française » qui se limite souvent au constat d'une administration omnipotente. La construction des frontières culturelles entre les groupes (Barth, 1969) au xix ${ }^{\mathrm{e}}$ siècle ne se comprend en effet qu'au prisme de la fabrique de l'État-nation, comme entité politique et culturelle, laquelle fabrique échappe pour partie, dans ses formes symboliques, aux pouvoirs publics. S'élabore ainsi une école nationale de pensée qui ne s'appuie pas sur les seuls canaux académiques (Bourdieu, 2000 ; Clément et al., 2006), mais se construit sur fond de rivalités entre agents privés et publics.

L'analyse du contenu des missions «musique » invite par ailleurs à relativiser la question des distances : l'altérité n'est pas seulement géographique, mais aussi temporelle et sociale. Plusieurs variables doivent être mobilisées et la formule «aller plus loin » dans une recherche recouvre plusieurs sens. Les obstacles à la connaissance des/sur/avec les « autres » sont enfin multiples. Aux traditionnels motifs économiques et techniques, s'ajoute ici l'inconscient culturel national dans sa forme processuelle, tel que pris dans des logiques de constructions identitaires internationale ${ }^{28}$ rivales, qui apparaît comme constituant la principale entrave à la fabrique d'un savoir sur l'altérité musicale.

\footnotetext{
${ }^{28}$ Les logiques nationales sont structurellement prises dans des logiques de différenciation culturelle internationale et transnationale, porteuses de « relations d'échange, de rivalité et de domination » (Heilbron, Guilhot, Jeanpierre, 2009, p. 123). Ainsi, la « culture française » au xix $\mathrm{e}^{\mathrm{e}}$ siècle se définit-elle relativement à d'autres cultures nationales allemande ou anglaise notamment (Heilbron, 2001, p. 143).
} 
Tableau 1. Inventaire des demandes de missions « musique » soumises au service des missions du ministère de l'Instruction publique (18421881)

\begin{tabular}{|c|c|c|c|c|}
\hline $\begin{array}{l}\text { Année } \\
\text { indemnité }\end{array}$ & Chargé de mission & Intitulé de la mission & \begin{tabular}{|l|} 
Destination et \\
type d'enquête
\end{tabular} & $\begin{array}{lr}\text { Champ } & \text { d'étude } \\
\text { spatial } & \text { et } \\
\text { temporel } & \end{array}$ \\
\hline $\begin{array}{l}1845 \text { Arrêté du } 10- \\
09300 \mathrm{fr}\end{array}$ & $\begin{array}{l}\text { Luzel, François-Marie (1821- } \\
1895)\end{array}$ & $\begin{array}{l}\text { Mission dans l'ancienne province de Bretagne à } \\
\text { l'effet d'y recueillir les chants et poésies populaires } \\
\text { en langue bretonne. }\end{array}$ & $\begin{array}{l}\text { France/ Bretagne } \\
\text {; Terrain }\end{array}$ & $\begin{array}{l}\text { France/ Bretagne } \\
\text { Contemporain }\end{array}$ \\
\hline \begin{tabular}{|l|}
1847 Arrêté du $28-$ \\
01 \\
$4000 \mathrm{fr}$
\end{tabular} & Danjou, Félix (1812-1866) & $\begin{array}{l}\text { Recherches scientifiques en Italie relatives aux } \\
\text { chants ecclésiastiques et aux chants populaires de } \\
\text { ce pays. }\end{array}$ & $\begin{array}{l}\text { Europe/ Italie } \\
\text { Bibliothèque }\end{array}$ & $\begin{array}{l}\text { Europe/ Italie } \\
\text { Haut Moyen- Âge }\end{array}$ \\
\hline 1848 Gratuite & $\begin{array}{l}\text { Laurent-de- Rillié, François- } \\
\text { Anatole (1828-1915) }\end{array}$ & $\begin{array}{l}\text { Mission autorisée par lettre du 27-09, pas d'arrêté } \\
\text { ni d'intitulé de mission. }\end{array}$ & $\begin{array}{l}\text { Europe/ Espagne } \\
\text {; Bibliothèque et } \\
\text { Terrain }\end{array}$ & $\begin{array}{l}\text { Europe/ Espagne ; } \\
\text { Haut Moyen-Âge à } \\
\text { Contemporain }\end{array}$ \\
\hline \begin{tabular}{|l|} 
Mission refusée en \\
février \\
Refusée
\end{tabular} & Clément, Félix (1822-1885) & $\begin{array}{l}\text { Mission ayant pour objet de former un recueil de } \\
\text { fac-similés des notations de la musique } \\
\text { ecclésiastique au Moyen-Âge. }\end{array}$ & $\begin{array}{l}\text { France } \\
\text { Bibliothèque }\end{array}$ & $\begin{array}{l}\text { France ; Moyen- } \\
\text { Âge }\end{array}$ \\
\hline$\left|\begin{array}{llr}1850-1851 & \text { Arrêté } \\
\text { du } & 28-1 & 1-1850 \\
\text { Arrêté } & \text { du } & 10-07- \\
1851 & 4 & 410 \mathrm{fr}\end{array}\right|$ & $\begin{array}{l}\text { L'abbé Normand Théodule- } \\
\text { Elzéar-Xavier (pseudonyme : } \\
\text { Nisard, Théodore) (1812-1888) } \\
\text { (pseudonyme : Nisard, } \\
\text { Théodore) (1812-1888) }\end{array}$ & $\begin{array}{l}\text { Mission scientifique ayant pour objet } \\
\text { la transcription à Montpellier et à St Gall en Suisse, } \\
\text { des Antiphonaires conservés dans ces deux villes ; } \\
\text { L'arrêté du 28-11- } 1850 \text { prévoit une double } \\
\text { mission en France et en Suisse. L'arrêté du 10-07- } \\
1851 \text { réduit ces deux missions à une seule, à } \\
\text { Montpellier. }\end{array}$ & $\begin{array}{l}\text { France/ } \\
\text { Montpellier } \\
\text { Bibliothèque }\end{array}$ & $\begin{array}{l}\text { Europe ; Moyen- } \\
\text { Âge }\end{array}$ \\
\hline $\begin{array}{l}1852 \text { Arrêté du } 19- \\
09 \text { Gratuite }\end{array}$ & $\begin{array}{l}\text { Baecker (de), Louis (1814- } \\
1896)\end{array}$ & $\begin{array}{l}\text { Mission en Allemagne à l'effet d'y rechercher } \\
\text { l'origine commune des chants populaires de ce pays } \\
\text { avec ceux du Nord de la France. }\end{array}$ & $\begin{array}{l}\text { Europe/ } \\
\text { Allemagne } \\
\text { Terrain }\end{array}$ & $\begin{array}{l}\text { France } \\
\text { Contemporain }\end{array}$ \\
\hline \begin{tabular}{|l|}
1852 Arrêté du $28-$ \\
10 \\
$1000 \mathrm{fr}$
\end{tabular} & Scudo, Paul (1806-1864) & $\begin{array}{l}\text { Mission ayant pour objet de recueillir des } \\
\text { documents relatifs à l'histoire de la musique en } \\
\text { France depuis le commencement du xixe siècle. }\end{array}$ & $\begin{array}{l}\text { Inconnue } \\
\text { Inconnu }\end{array}$ & France ; xixe siècle \\
\hline \begin{tabular}{|l|}
1855 Arrêté du 5- \\
04 \\
$1500 \mathrm{fr}$
\end{tabular} & $\begin{array}{l}\text { Hersart de la Villemarqué, } \\
\text { Théodore (1815-1895) }\end{array}$ & $\begin{array}{l}\text { Recherches dans les bibliothèques de manuscrits } \\
\text { intéressant l'histoire et la littérature des } \\
\text { départements de l'ouest de la France. }\end{array}$ & $\begin{array}{l}\text { Europe/ } \\
\text { Angleterre } \\
\text { Bibliothèque }\end{array}$ & $\begin{array}{l}\text { France ; Moyen- } \\
\text { Âge }\end{array}$ \\
\hline $\begin{array}{l}1856 \text { Arrêté du 20- } \\
02600 \mathrm{fr}\end{array}$ & Arbaud, Damase (1814-1876) & $\begin{array}{l}\text { Mission pour rechercher dans les bibliothèques du } \\
\text { midi de la France les chansons et les poésies } \\
\text { populaires qui seraient de nature à prendre place } \\
\text { dans le recueil préparé par les soins du Comité de } \\
\text { la langue, de l'histoire et des arts de la France. }\end{array}$ & $\begin{array}{l}\text { France/ } \\
\text { Midi } \\
\text { Bibliothèque }\end{array}$ & $\begin{array}{l}\text { France ; Moyen } \\
\text { Âge }\end{array}$ \\
\hline \begin{tabular}{|l|} 
Mission demandée \\
en 1861 , refusée en \\
janvier 1862
\end{tabular} & L’abbé Raillard ( ?- ?) & $\begin{array}{l}\text { Mission pour faire des recherches relatives à la } \\
\text { restauration du chant grégorien; Refusée }\end{array}$ & $\begin{array}{l}\text { France } \\
\text { et étranger } \\
\text { Bibliothèque }\end{array}$ & $\begin{array}{l}\text { Europe ; Moyen- } \\
\text { Âge }\end{array}$ \\
\hline$\left|\begin{array}{l}1865 \text { Arrêté du } 20- \\
04 \\
1500 \mathrm{fr}\end{array}\right|$ & Meyer, Paul (1840-1917) & $\begin{array}{l}\text { Mission littéraire en Angleterre, à l'effet d'y } \\
\text { recueillir des copies ou d'y prendre des collations } \\
\text { de diverses chansons de geste destinées au recueil } \\
\text { des anciens poètes de la France, publié sous les } \\
\text { auspices du ministère de l'Instruction publique. }\end{array}$ & $\begin{array}{l}\text { Europe/ } \\
\text { Angleterre } \\
\text { Bibliothèque }\end{array}$ & $\begin{array}{l}\text { France ; } \\
\text { Moyen-Âge }\end{array}$ \\
\hline $\begin{array}{l}1867 \text { Arrêté du 26- } \\
07700 \mathrm{fr}\end{array}$ & Meyer, Paul & $\begin{array}{l}\text { Mission à Oxford et Ashburnham-Palace pour y } \\
\text { continuer la copie et la collation de diverses } \\
\text { chansons de gestes destinées au recueil des anciens } \\
\text { poètes de la France, publié sous les auspices du } \\
\text { Ministère de l'instruction publique. }\end{array}$ & $\begin{array}{l}\text { Europe/ } \\
\text { Angleterre } \\
\text { Bibliothèque }\end{array}$ & $\begin{array}{l}\text { France ; Haut } \\
\text { Moyen-Âge }\end{array}$ \\
\hline $\begin{array}{l}1867 \text { Arrêté du 31- } \\
07600 \mathrm{fr}\end{array}$ & $\begin{array}{l}\text { Touroude, } \quad \text { Pierre-Parfait- } \\
\text { Arsène ( ?- ?) }\end{array}$ & $\begin{array}{l}\text { Mission en Belgique et en Hollande à l'effet d'y } \\
\text { recueillir les anciens chants et les poésies sacrées } \\
\text { de la Gaule. }\end{array}$ & $\begin{array}{l}\text { Europe/ Belgique } \\
\text { Hollande } \\
\text { Bibliothèque }\end{array}$ & $\begin{array}{l}\text { France ; Haut } \\
\text { Moyen-Âge }\end{array}$ \\
\hline$\left|\begin{array}{l}1868 \text { Arrêté du } 20- \\
10 \\
1200 \mathrm{fr}\end{array}\right|$ & Luzel, François-Marie & $\begin{array}{l}\text { Mission en Bretagne ayant pour objet } \\
\text { de recueillir les traditions orales pouvant servir à } \\
\text { l'étude comparée de l'histoire, de la philologie et } \\
\text { de la mythologie des différents peuples d'origine } \\
\text { celtique. }\end{array}$ & $\begin{array}{l}\text { France/ Bretagne } \\
\text { Terrain }\end{array}$ & $\begin{array}{l}\text { France } \\
\text { Contemporain }\end{array}$ \\
\hline $\begin{array}{ll}1870 \\
1200 \mathrm{fr}\end{array}$ & Clément, Félix & $\begin{array}{l}\text { Mission dans les bibliothèques du Midi de la } \\
\text { France pour rechercher les documents propres à } \\
\text { l'histoire de l'art musical. }\end{array}$ & $\begin{array}{l}\text { France/ } \\
\text { Midi } \\
\text { Bibliothèque }\end{array}$ & $\begin{array}{l}\text { Europe ; Moyen- } \\
\text { Âge }\end{array}$ \\
\hline \begin{tabular}{|l|}
1871 Arrêté du 5- \\
10 \\
$1000 \mathrm{fr}$
\end{tabular} & $\begin{array}{l}\text { Ruelle, Charles-Émile }(1833- \\
\text { 1912) }\end{array}$ & $\begin{array}{l}\text { Mission en Espagne à l'effet d'y recueillir dans les } \\
\text { bibliothèques de ce pays, les textes inédits relatifs } \\
\text { à la musique ancienne. }\end{array}$ & $\begin{array}{l}\text { Europe/ Espagne } \\
\text {; Bibliothèque }\end{array}$ & $\begin{array}{l}\text { Europe/ Grèce } \\
\text { Antiquité }\end{array}$ \\
\hline \begin{tabular}{|l|}
1872 Arrêté du 30- \\
03 \\
$1500 \mathrm{fr}$
\end{tabular} & $\begin{array}{l}\text { Dozon, Auguste (pseudonyme : } \\
\text { A. Argonne) (1822-1891) }\end{array}$ & $\begin{array}{l}\text { Mission dans le Rhodope pour y vérifier } \\
\text { l'authenticité des chants populaires de ce pays. }\end{array}$ & $\begin{array}{l}\text { Europe/ } \\
\text { Macédoine } \\
\text { Terrain }\end{array}$ & $\begin{array}{l}\text { Europe/ Macédoine } \\
\text {; Contemporain }\end{array}$ \\
\hline
\end{tabular}




\begin{tabular}{|c|c|c|c|c|}
\hline $\begin{array}{l}1874 \text { Arrêté du } 15- \\
09 \text { Gratuite }\end{array}$ & $\begin{array}{l}\text { Bourgault- Ducoudray, Louis- } \\
\text { Albert (1840-1910) }\end{array}$ & $\begin{array}{l}\text { Mission en Grèce pour continuer } \\
\text { ses études sur la musique des anciens et sur la } \\
\text { musique religieuse grecque. }\end{array}$ & $\begin{array}{l}\text { Europe/ Grèce } \\
\text { Bibliothèque }\end{array}$ & $\begin{array}{l}\text { Europe/ } \\
\text { Antiquité }\end{array}$ \\
\hline \begin{tabular}{|l|}
1880 Arrêté du 3- \\
05 \\
500 fr
\end{tabular} & Quellien, Narcisse (1848-1902) & $\mid \begin{array}{l}\text { Mission en Basse Bretagne ayant pour objet de } \\
\text { recueillir les mélodies populaires. }\end{array}$ & $\begin{array}{l}\text { France/ Bretagne } \\
\text {; Terrain }\end{array}$ & $\begin{array}{l}\text { France/ Bretagne } \\
\text { Contemporain }\end{array}$ \\
\hline \begin{tabular}{|l|}
881 Arrêté du 13- \\
05 \\
1000 fr
\end{tabular} & Quellien, Narcisse & $\mid \begin{array}{l}\text { Mission en Bretagne pour y recueillir } \\
\text { la musique et les paroles des chansons populaires. }\end{array}$ & $\begin{array}{l}\text { France/ Bretagne } \\
; \text { Terrain }\end{array}$ & $\begin{array}{l}\text { France/ Bretagne } \\
\text { Contemporain }\end{array}$ \\
\hline $\begin{array}{l}1881 \text { Arrêté du 17- } \\
06600 \text { fr }\end{array}$ & - $\begin{array}{l}\text { Bonnardot, François } \\
1926)\end{array}$ & $\begin{array}{l}\text { Mission à Turin, à l'effet d'exécuter dans la } \\
\text { bibliothèque de l'université de cette ville une copie } \\
\text { d'un manuscrit contenant la chanson de geste } \\
\text { d'Hervé de Metz. }\end{array}$ & $\begin{array}{l}\text { Europe/ Italie } \\
\text { Bibliothèque }\end{array}$ & $\begin{array}{l}\text { France } \quad ; \quad \text { Moyen- } \\
\text { Âge }\end{array}$ \\
\hline
\end{tabular}




\section{Références}

Anderson, B. (1996). L'imaginaire national : réflexions sur l'origine et l'essor du nationalisme (P. E. Dauzat, Trans.). Ed. La découverte.

Balteau, J., Barroux, M., Prevost, M., Tribout de Morembert, H., D'amat, R. \& Lobies, H. (1932). Dictionnaire de biographie française. Paris. Letouzey \& Ané.

Barth, F. (1969). Ethnic groups and boundaries: the social organization of culture difference. Boston. Little, Brown and Company.

Beaux-arts, M. de l'instruction publique et des (1890a). Table générale des archives des missions scientifiques et littéraires (vol. $3^{\mathrm{e}}$ série, T. XV bis). Paris. Ernest Leroux.

Beaux-arts, M. de l'instruction publique et des. (1890b). Table générale des archives des missions scientifiques et littéraires (vol. $3^{\mathrm{e}}$ série, T. XV bis). Paris. Ernest Leroux.

Bérose, L. (n.d.). Dictionnaire historique et biographique, disponible sur http:// www.berose.fr/document/spip.php?rubrique183

Bertho, C. (1980). «L'invention de la Bretagne ». Actes de La Recherche En Sciences Sociales, L’identité (35) : pp. 45-62.

Blanckaert, C. (n.d.). « Histoires du terrain. Entre savoirs et savoir-faire », dans Le terrain des sciences humaines xviii $e_{-\mathrm{xx}} e$ siècle (vol. 1996, pp. 9-55). Paris, Montréal. L'Harmattan.

Bourdieu, P. (1989). «Les conditions sociales de la circulation internationale des idées ». Actes de La Re-cherche En Sciences Sociales. La Circulation Internationale Des Idées (145) : pp. 38 .

Bourdieu, P. (2000). «L'inconscient d'école ». Actes de La Recherche En Sciences Sociales, Inconscients de l'école, pp. 3-5.

Bourguet, M. (1914). « Voyages et voyageurs », dans M. Delon (ed.), Dictionnaire européen des Lumières, pp. 1092-1095. Paris. PUF.

Bourguet, M.-N. (1996). «L'explorateur», dans M. Vovelle (ed.), L'homme des lumières, pp. 285-346. Paris. Le Seuil.

Bourquin, J.-C. (1993). L'État et les voyageurs savants. Légitimités individuelles et volontés politiques. Les missions du ministère de l'Instruction publique, 1840-1914. Université Paris ISorbonne.

Caron, F. (1997). Histoire des chemins de fer en France. Vol. 1 (1740-1883). Paris. Fayard.

Dubosclard, A., Grison, L. \& Jeanpierre, L. (2002). Entre rayonnement et réciprocité: contributions à l'histoire de la diplomatie culturelle. Paris. Publications de la Sorbonne. 
Fabre, D. \& Privat, J. M. (eds.). (2010). Savoirs romantiques, une naissance de l'ethnologie. Nancy. Presses universitaires.

Fétis, F.-J. (1860). Biographie universelle des musiciens et bibliographie générale de la musique, 1860-1868. Vols 8. Paris. Firmin-Didot.

Fétis, F.-J. (n.d.). Biographie universelle des musiciens et bibliographie générale de la musique, Supplément et complément 1878-1880. (A. Pougin, ed.). Paris. Firmin-Didot.

Guillet, F. (2003). «Entre stratégie sociale et quête érudite: les notables normands et la fabrication de la Normandie au xix ${ }^{\mathrm{e}}$ siècle ». Le Mouvement Social (203). pp. 89-111.

Heilbron, J. (2001). «Échanges culturels transnationaux et mondialisation : quelques réflexions ». Regards Sociologiques (22). pp. 141-154.

Heilbron, J. (2008). «Qu'est-ce qu'une tradition nationale en sciences sociales ? . Revue d'histoire des Sciences Humaines (18). pp. 3-16.

Heilbron, J., Guilhot, N. \& Jeanpierre, L. (2009). « Vers une histoire transnationale des sciences sociales ». Sociétés Contemporaines (173). pp. 121-145.

Heilbron, J., Lenoir, R. \& Sapiro, G. (eds.) (2004). Pour une histoire des sciences sociales : hommage à Pierre Bourdieu. Paris. Fayard.

Laborde, D. (2009). «Écrire une histoire universelle de la musique ? Procédures taxinomiques et aventures encyclopédiques ». En Désirs d'histoire. Politique, mémoire, identité. pp. 107-130. Paris, Turín, Budapest. L'Harmattan.

Latour, B. (2009). «Préface. Les moteurs immobiles de la mobilité », dans V. Guigueno \& M. Flonneau (eds.), De l'histoire des transports à l'histoire de la mobilité? État des lieux, enjeux et perspectives de re-cherche. pp. 7-10. Presses Universitaires de Rennes.

Le Goff, A. (2005). Ministère de l'Instruction publique, service des missions, missions scientifiques et littéraires ; Index nominatif des voyageurs et index géographique des destinations de leurs missions. Paris. Centre historique des archives nationales.

Le Goff, A. (2011). «Images et fantômes d'images dans les dossiers individuels des voyageurs dans les archives du Ministère de l'Instruction publique (xix $\mathrm{e}^{\mathrm{e}}$ siècle et début du $\mathrm{xx}^{\mathrm{e}}$ siècle) », dans C. Demelenaere-Douyère, M. Plouvier et C. Souchon (eds.), Des images et des mots : les documents figurés dans les archives. pp. 167-182. CTHS.

Le Goff, A. (n.d.). «Érudits de terrain et chercheurs d'aventures : les dossiers individuels des voyageurs dans les archives du ministère de l'Instruction publique (xix ${ }^{\mathrm{e}}$ siècle et début du $\mathrm{xx}^{\mathrm{e}}$ siècle) », dans T. Charmasson (ed.), Voyages et voyageurs. Sources pour l'histoire des voyages. pp. 51-68. Paris.CTHS.

Leterrier, S.-A. (1999). «Musique populaire et musique savante au xix e siècle. Du "peuple" au "public" ». Revue d'histoire du xix e Siècle, disponible sur http://rh19. revues.org/index 157.html 
Rey, A. (2010). « Lointain, aine », dans Dictionnaire historique de la langue française, pp. 1222. Paris. Robert.

Riviale, P. (1991). Un siècle d'archéologie au Pérou (1821-1914). Paris. L'Harmattan. Roche, D. (2003). Humeurs vagabondes : de la circulation des hommes et de l'utilité des voyages. Paris. Fayard.

Roche, F. \& Pigniau, B. (1995). Histoires de la diplomatie culturelle des origines à 1995. La Documentation française.

Sapiro, G. (ed.). (2009). L'espace intellectuel en Europe. De la formation des États- nations à la mondialisation xix ${ }^{e}$ siècle. Paris. La Découverte.

Studeny, C. (1995). L'invention de la vitesse : France, $\mathrm{xviii} e_{-\mathrm{xx}} e_{\text {siècle. Paris. Gallimard. }}$

Studeny, C. (2009). « La révolution des transports et l'accélération de la France (1770-1870) », pp. 117-139. En V. Guigueno et M. Flonneau (eds.), De l'histoire des transports à l'histoire de la mobilité ? État des lieux, enjeux et perspectives de recherche. pp. 117-139. Presses Universitaires de Rennes.

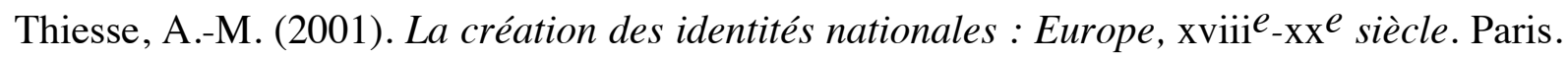
Le Seuil.

Vapereau, G. (1893). Dictionnaire universel des contemporains : contenant toutes les personnes notables de la France et des pays étrangers. Hachette.

Wiener, C. (2010). Voyage au Pérou et en Bolivie (1875-1877). Paris. Gingko. 